\title{
SORVETE COM ÔMEGA 3 E FARINHA DE JABUTICABA
}

\section{ICE CREAM WITH OMEGA 3 AND JABUTICABA FLOUR}

\author{
Joice Caetano da Silva Mussinato ${ }^{1}$, Frederico Lovato², Jussara Kowaleski ${ }^{3}$, Ricardo \\ Fiori Zara ${ }^{4}$, Daniela Miotto Bernardi ${ }^{*}$

\begin{abstract}
${ }^{1}$ Nutricionista, Centro Universitário Fundação Assis Gurgacz (FAG). ${ }^{2}$ Mestre em Processos Químicos e Biotecnológicos, Fundação para o Desenvolvimento Científico e Tecnológico (FUNDETEC). ${ }^{3}$ Mestre em Ciência e Tecnologia de Alimentos, Fundação para o Desenvolvimento Científico e Tecnológico (FUNDETEC), ${ }^{4}$ Doutor em química, Universidade Tecnológica Federal do Paraná (UTFPR), ${ }^{5}$ Nutricionista Doutora, Docente do Centro Universitário Fundação Assis Gurgacz (FAG), *Autor correspondente: danimiottober@gmail.com, https://orcid.org/0000-0001-9019-3835
\end{abstract}

DOI: $10.35984 /$ fjh.v2i2.214

\section{RESUMO}

O objetivo do trabalho foi desenvolver um sorvete com ômega 3 e farinha de casca de jabuticaba (FCJ), e avaliar as características físico químicas e sensoriais do produto. Foi avaliada a atividade antioxidante da FCJ e foram produzidas seis formulações de sorvete: FC (formulação controle), F1 (formulação com óleo de canola), F2 (formulação com óleo de canola $+2,5 \%$ de FCJ,) F3 (formulação com óleo de canola $+5 \%$ de FCJ), F4 (formulação com óleo de canola $+10 \%$ de FCJ) e F5 (formulação com óleo de canola $+15 \%$ de FCJ). Os sorvetes foram submetidos às análises físico-químicas e sensoriais. A FCJ apresentou atividade antioxidante de $4081,50 \mathrm{ug} / \mathrm{g}$ equivalente a ácido gálico. O sorvete apresentou excelente composição nutricional. A incorporação de óleo de canola não prejudicou a aceitabilidade dos produtos, porém as maiores concentrações de FJ prejudicaram a aceitabilidade da amostra. Concluiu-se que o sorvete desenvolvido teve boa aceitabilidade e características nutricionais o que confere potencial de mercado ao produto.

Palavras-chave: Antioxidante, casca de jabuticaba, ácido alfa-linolênico.

\begin{abstract}
The objective of the work was to develop an ice cream with omega 3 and jabuticaba peel flour (JPF), and evaluate the physical chemical and sensory characteristics of the product. The antioxidant activity of JPF was evaluated and six ice cream formulations were produced: FC (control formulation), F1 (formulation with canola oil), F2 (formulation with canola oil $+2.5 \%$ JPF) F3 (formulation with canola oil $+5 \%$ JPF), F4 (formulation with oil canola $+10 \% \mathrm{JPF}$ ) and F5 (formulation with canola oil $+15 \%$ JPF). The ice creams were subjected to physicalchemical and sensory analyzes. JPF showed antioxidant activity of $4081.50 \mathrm{ug} / \mathrm{g}$ equivalent to gallic acid. The ice cream showed excellent nutritional composition. The incorporation of canola oil did not impair the acceptability of the products, but the higher concentrations of JPF impaired the acceptability of the ice cream. It was concluded that the developed ice cream had good acceptability and nutritional characteristics, which gives the product a market potential.
\end{abstract}

Keywords: Antioxidant, jabuticaba peel, alpha-linolenic acid.

\section{INTRODUÇÃO}

Na contemporaneidade as mudanças nos hábitos alimentares se tornam cada dia mais pertinentes para a população brasileira, não basta apenas alimentar-se, é necessário consumir alimentos que possam trazer benefícios a saúde. Neste contexto, desenvolver um produto alimentício que una essas duas características, e, que 
possua um bom tempo de prateleira, sem prejudicar as características nutricionais e sensoriais, é desejável para o consumidor (MADI, 2010; MORAES, 2006).

Nos alimentos, os radicais livres provocam oxidações que podem ser prejudiciais à saúde, promovendo a degradação oxidativa dos lipídios, vitaminas e pigmentos, levando a diminuição do valor nutritivo e tempo de prateleira, portanto, se for acrescentado um ingrediente antioxidante a uma determinada formulação o produto pode apresentar benefícios em ambas questões, durabilidade e funcionalidade fisiológica (BOROSKI et al. 2015; FERRARI,1998).

A jabuticaba é uma fruta muito comum na região sul do país, seu sabor adocicado faz com que seja uma fruta muito apreciada, ela se constitui em casca, bagaço e semente. A jabuticaba está ganhando destaque na indústria de alimentos devido à composição da casca, seu subproduto, a qual possui um antioxidante natural e eficaz devido aos elevados teores de compostos fenólicos, além disso, a casca também é fonte de fibras, as quais podem contribuir para o enriquecimento de uma preparação (ZAGO,2014; LAMOUNIER, 2015).

O óleo de canola é pouco usado na dieta dos brasileiros, porém pode ser considerado um aliado na manutenção da saúde do organismo humano, além de ser um produto de fácil acesso e de custo relativamente baixo. A canola, assim como outras oleaginosas, da mesma maneira que a linhaça e a soja, fazem parte de produtos considerados fontes de ácido $\alpha$-linolênico (ALA), o qual é um ácido graxo (AG) essencial precursor dos demais (AG) da família ômega 3. Entre os benefícios do ALA, pode-se citar que ele auxilia na redução do colesterol LDL e em alguns casos aumenta o colesterol HDL (NOVELLO, 2010; PITA, 2006). Sorvetes são considerados preparações obtidas através da mistura de leite, derivados do leite, frutas, açúcares, proteínas, óleos, gorduras e qualquer outro produto alimentício, desde que o mesmo não descaracterize o sorvete (ANVISA, 2008). Por se tratar de uma preparação de boa aceitabilidade em todas as idades, e por conter diversas variações de sabores, se torna um produto de fácil manipulação e atrativo para inovações e experimentos na área da tecnologia de alimentos (SOUZA, 2010).

Dessa forma, associar um produto muito consumido no país a uma formulação inovadora com benefícios para saúde de seus consumidores, é um dos objetivos do estudo. Bem como, determinar o potencial antioxidante da farinha da casca de jabuticaba (FCJ), elaborar formulações de sorvete com a FCJ, bem como incorporar o óleo de canola rico em ômega 3, e avaliar as características sensoriais e físicoquímicas do produto, é a finalidade do trabalho.

\section{METODOLOGIA}

\subsection{Matéria Prima}

A farinha de jabuticaba (Myrciaria cauliflora), foi adquirida por meio de doação realizada por uma fazenda da região oeste do Paraná. O processo se iniciou com a higienização das jabuticabas, na primeira etapa, elas foram lavadas em água corrente, em seguida imersas em solução clorada a 200 ppm por quinze minutos, logo depois foram despolpadas, suas cascas higienizadas. Após este processo, foi conduzida a secagem para a obtenção da farinha de jabuticaba, a qual foi feita em estufa de circulação de ar (marca: Labstore, modelo: serie $400 \mathrm{MB}$ ) em temperatura de $40^{\circ} \mathrm{C}$ por 72 horas, posteriormente o material foi triturado no liquidificador (marca: Victory), e peneirado em malha de três centímetros, em seguida realizou-se o armazenamento em potes plásticos até o momento da sua utilização. 
Os demais ingredientes, nata, creme de leite, açúcar, essência de baunilha, liga neutra e o óleo de canola, utilizados na elaboração das formulações dos sorvetes foram adquiridos no comércio local.

\subsection{Formulações de sorvete}

Foram desenvolvidas seis formulações de sorvete, cujos ingredientes e as quantidades utilizadas estão indicados na Tabela 1, sendo: FC (formulação controle), F1 (formulação com óleo de canola), F2 (formulação com óleo de canola $+2,5 \%$ de farinha de casca de jabuticaba) F3 (formulação com óleo de canola $+5 \%$ de farinha de casca de jabuticaba), F4 (formulação com óleo de canola $+10 \%$ de farinha de casca de jabuticaba) e F5 (formulação com óleo de canola $+15 \%$ de farinha de casca de jabuticaba).

Tabela 1. Formulações das amostras de sorvete com incorporação de óleo de canola e farinha de casca de jabuticaba (FCJ).

\begin{tabular}{lcccccc}
\hline Ingredientes & FC & F1 & F2 & F3 & F4 & F5 \\
\hline Creme de leite light & $*$ & 28,0 & 27,6 & 27,2 & 26,8 & 26,5 \\
& & $*$ & $*$ & $*$ & $*$ & $*$ \\
Nata & 31,4 & $*$ & $*$ & $*$ & $*$ & \\
logurte natural desnatado & 50,0 & 44,8 & 44,1 & 43,5 & 43,0 & 42,4 \\
Açúcar refinado & 15,6 & 14,0 & 14,0 & 13,6 & 13,4 & 13,2 \\
Liga neutra & 1,5 & 1,4 & 1,4 & 1,3 & 1,34 & 1,3 \\
Farinha de casca de jabuticaba (FCJ) & $*$ & $*$ & 1,4 & 2,7 & 4,0 & 5,3 \\
Óleo de canola & $*$ & 10 & 10 & 10 & 10 & 10 \\
Essência de baunilha & 1,5 & 1,4 & 1,38 & 1,3 & 1,34 & 1,3 \\
\hline
\end{tabular}

FC (formulação controle), F1 (formulação com óleo de canola), F2 (formulação com óleo de canola + 2,5\% de FCJ,) F3 (formulação com óleo de canola + 5\% de FCJ), F4 (formulação com óleo de canola $+10 \%$ de FCJ) e F5 (formulação com óleo de canola + $15 \%$ de FCJ)

Para o preparo dos sorvetes iniciou-se com a higienização das bancadas com álcool $70 \%$. Os utensílios utilizados, como batedeira, colheres e bacias foram submersos em água clorada a 200 ppm por 15 minutos, a sorveteira utilizada para 0 processo de finalização do sorvete também foi higienizada com álcool $70 \%$, para garantir a assepsia necessária, não ocorrendo riscos de contaminação.

O processo de fabricação do sorvete se iniciou com a pesagem das respectivas proporções (Tabela 01) e colocados na batedeira. Após sua homogeneização na batedeira, as preparações foram encaminhadas uma a uma, separadamente para a sorveteira, onde a preparação adquiriu a consistência de sorvete, em seguida os sorvetes foram acondicionados em potes plásticos no freezer até o momento da análise sensorial, o fluxograma de processamento está apresentado na Figura 01.

\subsection{Análises Físico-Químicas e sensorial}

Foi realizada a análise de fenóis totais na farinha de casca de Jabuticaba de acordo com os métodos descritos por Boroski et al. (2015). As análises da composição centesimal da farinha de jabuticaba e dos sorvetes foram conduzidas de acordo com o Manual do Instituto Adolfo Lutz (2008) com as metodologias de proteínas (037/IV), lipídeos (032/IV), cinzas (018/IV), umidade (0,12/IV) e carboidratos determinados por diferença. As quantidades totais de ácidos graxos ômega 3 e ômega 6 foram determinadas por cálculo nutricional segundo os critérios estabelecidos na Resolução RDC $n^{\circ} 360$, de 23 de dezembro de 2003, sendo utilizado a tabela Brasileira de Composição de Alimentos (TACO, 2011) como referência para o cálculo. 

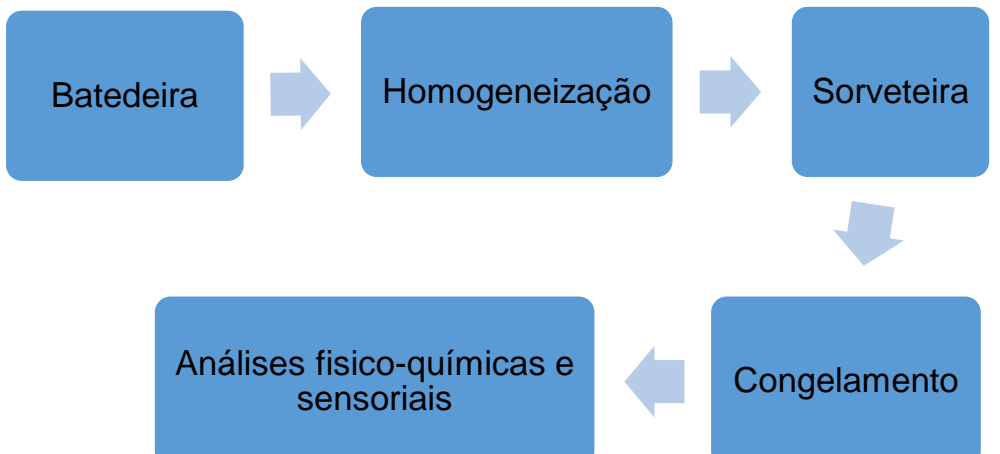

Congelamento

Figura 1. Fluxograma geral do processamento do sorvete com ômega 3 e farinha de casca de jabuticaba.

O projeto de pesquisa foi encaminhado para a o comitê de ética em pesquisa com humanos, onde teve parecer favorável $\left(n^{\circ} 2.617 .581\right)$ para o desenvolvimento da pesquisa. A análise sensorial procedeu da seguinte forma: os voluntários foram selecionados em um centro universitário do Oeste Paraná, em que assinaram o termo de consentimento livre e esclarecido. As degustações aconteceram no laboratório de nutrição em cabines específicas para as análises. Os voluntários receberam $10 \mathrm{~g}$ de sorvete de cada amostra, as mesmas foram servidas em copos descartáveis identificados com números aleatórios de três dígitos, em uma temperatura à $-4^{\circ} \mathrm{C}$, junto com um copo de água para retirada do sabor da amostra anterior. Os sorvetes foram servidos de forma monádica em blocos completos aleatorizados (MACFIE et al, 1989). A aceitabilidade foi determinada por escalas, sendo que para aceitação global, aparência, aroma, sabor e textura foi utilizada uma escala hedônica estruturada de nove pontos, que correspondiam a 9 - Gostei muitíssimo; 8 - Gostei muito; 7 - Gostei moderadamente; 6 - Gostei ligeiramente; 5 - Não gostei nem desgostei; 4 - Desgostei ligeiramente; 3 - Desgostei moderadamente; 2 - Desgostei muito; 1 - Desgostei muitíssimo. A intenção de compra foi determinada por uma escala estruturada de cinco pontos, os quais correspondiam a 5 - Certamente compraria; 4- Provavelmente compraria; 3- Tenho dúvida se compraria ou não; 2- provavelmente não compraria 1 Certamente não compraria (DUTCOSKY, 2013).

Os dados referentes a análise sensorial das seis amostras foram submetidos à análise da variância (ANOVA). Em seguida, utilizou-se o teste de Tukey, em nível de significância de $5 \%(p<0,05)$, para identificar a diferença entre as formulações, em um programa Microsoft Excel.

\section{RESULTADOS E DISCUSSÃO}

\subsection{Composição química}

Os testes de fenóis totais na farinha de casca de jabuticaba indicaram uma concentração de 4081,50ug/g equivalente a ácido gálico em cada $100 \mathrm{~g}$ de amostra. Compostos fenólicos apresentam importante atividade antioxidante, sendo esta benéfica tanto para quem consome o produto, pois os compostos fenólicos atuam como um antioxidante natural no organismo humano, bem como um benefício para o produto, que ganha tempo de prateleira mediante um composto que retarda a oxidação lipídica, trabalhando como um antioxidante natural (Bernardi et al., 2016). A proteção antioxidante da FCJ é particularmente interessante para o sorvete desenvolvido neste experimento, pois o mesmo possui incorporação de óleo de 
canola, reconhecida fonte de ácido graxo insaturado do tipo alfa-linolênico, o qual possui menor estabilidade oxidativa.

$\mathrm{Na}$ Tabela 2 estão apresentados os valores da composição centesimal da farinha de jabuticaba e dos sorvetes produzidos. É possível verificar que a farinha de casca de jabuticaba apresenta elevados teores de carboidratos e quantidades relevantes de proteínas, lipídios e cinzas. Em relação às formulações de sorvete, a incorporação de óleo de canola (F1, F2, F3, F4, F5) e de farinha de jabuticaba (F2, F3, F4, F5) não promoveram modificações intensas na composição do produto quando comparados à formulação controle (FC). Segundo dados da Tabela de Composição de Alimentos IBGE (2011), um sorvete industrializado contém em $100 \mathrm{~g}$ de produto o equivalente a 25,13 gramas de carboidrato 3,60 gramas de proteína, 11 gramas de lipídeo e 0,87 gramas de fibras. Neste sentido é possível observar que as formulações de sorvete produzidas apresentaram boa característica nutricional.

Tabela 2. Composição centesimal da farinha de casca de jabuticaba e das formulações de sorvete com incorporação de ômega 3 (óleo de canola) e farinha de casca de jabuticaba

\begin{tabular}{lrrrrrrr} 
& FCJ & \multicolumn{7}{c}{ Formulações de sorvetes } \\
Nutrientes & & \multicolumn{1}{c}{ FC } & \multicolumn{1}{c}{ F1 } & \multicolumn{1}{c}{ F2 } & \multicolumn{1}{c}{ F3 } & \multicolumn{1}{c}{ F4 } & \multicolumn{1}{c}{ F5 } \\
\hline Umidade & 12,6 & 62,83 & 61,99 & 60,96 & 61,04 & 62,23 & 61,33 \\
Cinzas & 4,25 & 0,95 & 0,99 & 1,00 & 1,06 & 0,98 & 1,09 \\
Proteínas & 5,18 & 3,42 & 3,22 & 2,42 & 3,16 & 3,24 & 3,32 \\
Lipídios & 5,22 & 0,57 & 0,57 & 0,47 & 0,43 & 0,50 & 0,53 \\
Carboidrato (por diferença) & 72,74 & 32,23 & 33,23 & 35,15 & 34,31 & 33,05 & 33,73 \\
\hline
\end{tabular}

FCJ (Farinha de casca de jabuticaba. FC (formulação controle), F1 (formulação com óleo de canola), F2 (formulação com óleo de canola $+2,5 \%$ de FCJ,) F3 (formulação com óleo de canola $+5 \%$ de FCJ), F4 (formulação com óleo de canola $+10 \%$ de FCJ) e F5 (formulação com óleo de canola $+15 \%$ de FCJ)

Com relação a incorporação do ômega 3 e ômega 6 nas formulações os valores calculados encontram-se na Tabela 3. Os teores calculados de ômega 3, mostraram que o sorvete produzido pode ser uma importante fonte desta família de ácidos graxos. Dados semelhantes foram encontrados em estudos realizados por De Pinho et al. (2015), que na sua formulação de sorvete foi incorporada amêndoa de baru rica em ômega 3 e ômega 9 , para ele a ingestão de $20 \mathrm{~g}$ de sua formulação atenderia de 40 a $65 \%$ da recomendação diária de ácido linoleico para uma criança de 4 a 8 anos. Em estudos de Backes (2013), a incorporação de 30\% e 15\% de óleo de canola em formulações de salames mostrou que os provadores não perceberam diferença na aceitabilidade dos produtos em relação a formulação controle isenta de óleo de canola. Rodrigues da Silva Cechin e Bernardi (2020) também verificaram quantidades semelhantes de ômega 3 em formulações de patê de frango com incorporação de óleo de canola.

Tabela 3. Conteúdo calculado de ômega 3 total e de ômega 6 total em $100 \mathrm{~g}$ das formulações de sorvete com incorporação de ômega 3 (óleo de canola) e farinha de casca de jabuticaba

\begin{tabular}{lrccccc}
\hline Família de ácido graxo & \multicolumn{5}{c}{ Formulações de sorvetes } \\
& $\mathrm{FC}$ & $\mathrm{F} 1$ & $\mathrm{~F} 2$ & $\mathrm{~F} 3$ & $\mathrm{~F} 4$ & $\mathrm{~F} 5$ \\
\hline Ômega 3 & 0 & $2,17 \mathrm{~g}$ & $2,17 \mathrm{~g}$ & $2,17 \mathrm{~g}$ & $2,17 \mathrm{~g}$ & $2,17 \mathrm{~g}$ \\
Ômega 6 & 0 & $6,3 \mathrm{~g}$ & $6,3 \mathrm{~g}$ & $6,3 \mathrm{~g}$ & $6,3 \mathrm{~g}$ & $6,3 \mathrm{~g}$ \\
\hline
\end{tabular}

FC (formulação controle), F1 (formulação com óleo de canola), F2 (formulação com óleo de canola + $2,5 \%$ de Farinha de casca de jabuticaba - FCJ) F3 (formulação com óleo de canola + 5\% de FCJ), F4 (formulação com óleo de canola $+10 \%$ de FCJ) e F5 (formulação com óleo de canola + 15\% de FCJ) 


\subsection{Análise Sensorial}

Participaram da análise sensorial 114 degustadores não treinados, de ambos os sexos, com idade dentre 18 e 38 anos. Em relação ao registro de avaliação sensorial sobre os atributos aceitação global, aparência, aroma, sabor e textura para FC, F1, F2, F3, F4, F5, FW6 os resultados estão apontados na Tabela 4.

Tabela 4. Aceitabilidade das formulações de sorvete com incorporação de ômega 3 (óleo de canola) e farinha de casca de jabuticaba

\begin{tabular}{|c|c|c|c|c|c|c|c|c|}
\hline \multirow{2}{*}{$\begin{array}{l}\text { Atributos } \\
\text { sensoriais }\end{array}$} & \multicolumn{6}{|c|}{ Formulações de sorvetes } & \multirow[t]{2}{*}{ DMS } & \multirow[t]{2}{*}{ Valor $\mathbf{P}$} \\
\hline & FC & $\mathrm{F} 1$ & F2 & F3 & F4 & F5 & & \\
\hline $\begin{array}{l}\text { Aceitação } \\
\text { Global }\end{array}$ & $\begin{array}{l}7,78 \pm \\
1,53 \mathrm{a}\end{array}$ & $\begin{array}{l}7,42 \pm \\
1,66 \mathrm{a}\end{array}$ & $\begin{array}{l}7,17 \pm \\
1,66 a b\end{array}$ & $\begin{array}{l}6,72 \pm \\
1,89 \mathrm{bc}\end{array}$ & $\begin{array}{l}6,75 \pm \\
1,85 b c\end{array}$ & $\begin{array}{c}6,23 \pm \\
1,95 \mathrm{c}\end{array}$ & 0,591 & $<0,001$ \\
\hline Aparência & $\begin{array}{l}7,68 \pm \\
1,74 \mathrm{a}\end{array}$ & $\begin{array}{l}7,18 \pm \\
1,93 \mathrm{ac}\end{array}$ & $\begin{array}{l}7,18 \pm \\
1,93 a c\end{array}$ & $\begin{array}{l}6,99 \pm \\
1,78 \mathrm{ac}\end{array}$ & $\begin{array}{l}6,74 \pm \\
1,96 \mathrm{bc}\end{array}$ & $\begin{array}{l}6,62 \pm \\
2,03 b\end{array}$ & 0,649 & $<0,001$ \\
\hline Aroma & $\begin{array}{c}7,63 \pm \\
1,67^{\underline{a}}\end{array}$ & $\begin{array}{l}7,26 \pm \\
1,84 \mathrm{ac}\end{array}$ & $\begin{array}{l}7,16 \pm \\
1,64 \mathrm{ac}\end{array}$ & $\begin{array}{l}6,85 \pm \\
1,82 \mathrm{bc}\end{array}$ & $\begin{array}{l}6,92 \pm \\
1,70 \mathrm{bc}\end{array}$ & $\begin{array}{c}6,53 \pm \\
1,84 c\end{array}$ & 0,592 & $<0,001$ \\
\hline Sabor & $\begin{array}{c}7,82 \pm \\
1,62^{\mathrm{a}}\end{array}$ & $\begin{array}{l}7,35 \pm \\
1,90 \mathrm{ac}\end{array}$ & $\begin{array}{l}7,03 \pm \\
2,03 \mathrm{bc}\end{array}$ & $\begin{array}{l}6,72 \pm \\
2,02 b c\end{array}$ & $\begin{array}{l}6,66 \pm \\
2,10 \mathrm{bc}\end{array}$ & $\begin{array}{l}5,92 \pm \\
2,30 d\end{array}$ & 0,699 & $<0,001$ \\
\hline Textura & $\begin{array}{c}7,64 \pm \\
1,81^{\mathrm{a}}\end{array}$ & $\begin{array}{l}7,36 \pm \\
1,97 \mathrm{ac}\end{array}$ & $\begin{array}{l}6,88 \pm \\
2,10 \mathrm{ad}\end{array}$ & $\begin{array}{l}6,90 \pm \\
2,03 b c\end{array}$ & $\begin{array}{l}6,84 \pm \\
1,87 \mathrm{bd}\end{array}$ & $\begin{array}{l}6,41 \pm \\
2,03 d\end{array}$ & 0,704 & $<0,001$ \\
\hline $\begin{array}{l}\text { Intenção de } \\
\text { compra }\end{array}$ & $\begin{array}{l}4,28 \pm \\
0,90 a\end{array}$ & $\begin{array}{l}4,03 \pm \\
1,00 \mathrm{ac}\end{array}$ & $\begin{array}{l}3,91 \pm \\
1,01 \mathrm{ad}\end{array}$ & $\begin{array}{c}3,64 \pm 1,19 b \\
c d\end{array}$ & $\begin{array}{c}3,56 \pm 1,1 \\
8 \mathrm{bd}\end{array}$ & $\begin{array}{c}3,33 \pm 1 \\
, 30 \mathrm{~b}\end{array}$ & 0,396 & $<0,001$ \\
\hline
\end{tabular}

FC (formulação controle), F1 (formulação com óleo de canola), F2 (formulação com óleo de canola + 2,5\% de Farinha de casca de jabuticaba - FCJ) F3 (formulação com óleo de canola + 5\% de FCJ), F4 (formulação com óleo de canola + 10\% de FCJ) e F5 (formulação com óleo de canola + 15\% de FCJ). DMS (diferença mínima significativa).

A aceitabilidade do produto foi bem-sucedida, uma vez que as médias de aceitabilidade dos produtos na maioria dos atributos avaliados ficaram igual ou superior à 6 ("gostei ligeiramente"). Do ponto de vista estatístico, as amostras diferiram estatisticamente em relação a todos os atributos avaliados. De maneira geral as amostras FC, F1 e F2 apresentaram aceitabilidade similar estatisticamente, isso traz duas conclusões importantes, a primeira é que a incorporação de óleo de canola, como fonte de ômega 3, não prejudicou a aceitabilidade do produto, a segunda é que a incorporação de pequenas concentrações de farinha de casca jabuticaba, como fonte de antioxidante, não prejudica a aceitabilidade do produto. Também se verificou que as maiores incorporações de farinha de casca de jabuticaba (F3, F4, F5) afetaram negativamente a aceitabilidade do produto, sendo as menores medias de aceitação a medida que a concentração da farinha de jabuticaba aumenta.

Resultados semelhantes foram obtidos por Rodrigues da Silva Cechin e Bernardi (2020) que também observaram que a incorporação de óleo de canola em formulações de patê não prejudicou a aceitabilidade do produto, ao passo que, a incorporação de farinha de bagaço de uva (como antioxidante natural) prejudicou a aceitabilidade do patê. Por outro lado, Lamounier et al. (2015) desenvolveu formulações de sorvetes enriquecidos com FCJ em sua análise sensorial a aceitabilidade da formulação FC (sem adição de FCJ) e a Formulação com $5 \%$ de incorporação de FCJ não sofreram diferenciação nas avaliações de textura, sabor, 
aparência e aspecto global entre os participantes, confirmando mais uma vez que a adição em concentrações menores de FCJ não interfere na aceitabilidade do produto.

\section{CONSIDERAÇÕES FINAIS}

Os dados obtidos neste estudo confirmam a farinha de casca de jabuticaba como uma importante fonte de compostos fenólicos e a incorporação desta farinha associada ao óleo de canola em formulações de sorvete apresenta-se como uma estratégia benéfica do pronto de vista nutricional e tecnológico, pois o produto passa a apresentar ômega 3 e antioxidantes naturais. Em relação à análise sensorial foi possível verificar que o provador aceitou bem a incorporação do óleo de canola e de pequenas concentrações da farinha de jabuticaba. $O$ desenvolvimento de produtos inovadores e com melhores características nutricionais é uma tendência no mercado, neste sentido o sorvete desenvolvido neste estudo apresenta-se como uma alternativa interessante para a indústria de alimentos que busca saudabilidade, pois ao mesmo tempo o produto apresenta ômega 3 e antioxidantes naturais.

\section{REFERÊNCIAS}

ARAÚJO, C. R. R. Composição química, potencial oxidante e hipolipidêmico da farinha da casca de Myrciaria couliflora (jabuticaba). Diamantina, 2011. $119 \mathrm{f}$. Dissertação (Mestrado em Química Orgânica) - Universidade Federal dos Vales do Jequitinhonha e Mucuri. Diamantina - Minas Gerais, 2011.

BACKES, A. M. et al. Características físico-químicas e aceitação sensorial de salame tipo Italiano com adição de óleo de canola. Semina: Ciências Agrárias, v. 34, n. 2, 2013. https://doi.org/10.5433/1679-0359.2013v34n6Supl2p3709

BERNARDI, D. M. et al. Ômega-3 in meat products: benefits and effects on lipid oxidative stability. J Sci Food Agric, v. 96, n.8, p.2620-34, 2016. https://doi.org/10.1002/jsfa.7559.

BOROSKI, M.; VISENTAINER, J. V.; COTTICA, S. M.; MORAIS, D. R. Antioxidantes. Princípios e métodos analíticos. Curitiba, Appris, 2015, p. 49 - 61.

DUTCOSKY, S. D. Análise sensorial de alimentos. 4 ed. rev. e. ampl. - Curitiba PR. Editora Champagnat, 2013.

DE PINHO, Lucinéia et al. Enriquecimento de sorvete com amêndoa de baru (Dipteryx Alata Vogel) e aceitabilidade por consumidores. Unimontes Científica, v. 17, n. 1, p. 39-49, 2015.

FERRARI, Carlos Kusano Bucalen. Oxidação lipídica em alimentos e sistemas biológicos: mecanismos gerais e implicações nutricionais ep mecanismos gerais e implicações nutricionais e patológicas tológicas. 1998.

LAMOUNIER, Marina Leopoldina et al. Desenvolvimento e caracterização de diferentes formulações de sorvetes enriquecidos com farinha da casca da jabuticaba 
(Myrciaria cauliflora). Revista do Instituto de Laticínios Cândido Tostes, v. 70, n. 2, p. 93-104, 2015. https://doi.org/10.14295/2238-6416.v70i2.400

MADI, Luis; COSTA, ACPB; REGO, Raul Amaral. Brasil Food trends 2020. São Paulo: FIESP: ITAL, 2010.

MORAES, Fernanda P., and Luciane M. Colla. "Alimentos funcionais e nutracêuticos: definições, legislação e benefícios à saúde." Revista eletrônica de farmácia 3.2 (2006): 109-122.

MONTEIRO, A. R. G. Introdução à análise sensorial de alimentos. Coleção Fundamentum. Editora da Universidade Estadual de Maringá, Maringá, 2005.

NOVELLO, D; FRANCESCHINI, P.; QUINTILIANO, D. A. A importância dos ácidos graxos ômega-3 e ômega-6 para a prevenção de doenças e na saúde humana. Revista Salus, v. 2, n. 1, 2010.

PITA, M. C. G. et al. Efeito da suplementação de linhaça, óleo de canola e vitamina E na dieta sobre as concentrações de ácidos graxos poliinsaturados em ovos de galinha. Arquivo Brasileiro de Medicina Veterinária e Zootecnia, v. 58, n. 5, p. 925931, 2006. Doi: https://doi.org/10.1590/S0102-09352006000500031.

RODRIGUES DA SILVA CECHIN, K.; BERNARDI, D. Elaboração e análise sensorial de patê de frango com ômega-3 e antioxidantes naturais do bagaço de uva. FAG Journal of Health (FJH), v. 2, n. 1, p. 93-102, 2020.

https://doi.org/10.35984/fjh.v2i1.139

SOUZA, Jean CB et al. Sorvete: composição, processamento e viabilidade da adição de probiótico. Alimentos e Nutrição Araraquara, v. 21, n. 1, p. 155-165, 2010.

ZAGO, M. F. C. et al. Aproveitamento de resíduo agroindustrial de jabuticaba no desenvolvimento de formulação de cookie para a alimentação escolar. 2014. 